\title{
Highly efficient Förster resonance energy transfer between CdTe nanocrystals and two different dye molecules
}

\author{
E. Alphandéry ${ }^{* a}$, L. M. Walsh ${ }^{\text {a }}$, Y. Rakovich ${ }^{\mathrm{a}}$, A. L. Bradley ${ }^{\mathrm{a}}$, J. F. Donegan ${ }^{\mathrm{a}}$, N. Gaponik ${ }^{\mathrm{b}}$, Y.

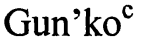 \\ ${ }^{a}$ Semiconductor Photonics Group, Physics Department, Trinity College, Dublin 2, Ireland; \\ ${ }^{\mathrm{b}}$ Institute of Physical Chemistry, University of Hamburg, 20146 Hamburg, Germany; \\ ${ }^{\mathrm{c}}$ Chemistry Department, Trinity College, Dublin 2, Ireland
}

\begin{abstract}
We report highly efficient Förster resonance energy transfer between CdTe nanocrystals and two different dyes, Rhodamine B and Oxazine, where the nanocrystals are mixed with the dyes on top of glass substrates. A faster NC decay curve is observed in the samples containing NCs mixed with dyes than in those containing NCs on their own. For the samples containing nanocrystals mixed with Rhodamine B, room temperature PL measurements are presented as a function of the ratio between the amount of acceptors and the amount of donors, $C_{A} / C_{D}$. This ratio is varied between 0.03 and 5. The strongest enhancement of the acceptor PL intensity relative to that of the donor PL intensity is reached for $0.2<C_{A} / C_{D}<5$, suggesting that most efficient FRET is also achieved in this region.
\end{abstract}

Keywords: CdTe nanocrystals, Rhodamine B, Oxazine, FRET.

\section{INTRODUCTION}

Förster resonance energy transfer (FRET) is a non-radiative dipole-dipole interaction, which results in the transfer of energy from one molecule, called a donor, to another molecule, called an acceptor. In order for FRET to occur, the following three conditions have to be fulfilled. The distance between donors and acceptors has to be less than twice the Förster distance $\left(2 \mathrm{R}_{0}\right)$, the absorption spectrum of the acceptor has to overlap with the emission spectrum of the donor and the energy of the donor emission has to be above that of the acceptor absorption. ${ }^{1,2}$ In terms of applications, FRET can be used to measure the distance between a donor and an acceptor. The latter is deduced from an estimate of the FRET efficiency, which is made either through lifetime or continuous wave PL measurements. ${ }^{1,2}$ Recently, the introduction of nanocrystals (NCs) has led to new and more efficient FRET pairs ${ }^{3,4}$ than those composed of dyes of different species. In addition, the conjugation of these pairs to proteins has been achieved, paving the way for more sensitive FRET measurements with biological samples.

In this paper, we study the mechanism of energy transfer between CdTe NCs (donors) and two different types of dyes (acceptors), Rhodamine B (RhB) or Oxazine. The samples are prepared following the technique of the mixed solid films. With this technique, the dyes and nanocrystals are expected to be in close proximity ${ }^{5}(<10 \mathrm{~nm})$, fulfilling one of the conditions required for FRET. The nanocrystals are introduced in the FRET pair in order to enhance the FRET efficiency. This can be achieved for two reasons. Firstly, the NCs have a high quantum yield (>15\%). Secondly, the position of the NC PL peak can be adjusted in order to produce maximum spectral overlap between the NC PL peak and the dye absorption spectrum. Other advantages of the FRET pairs studied in this paper are presented in ref. 1. With regard to the two different dyes studied here, the following remarks should be made. Prepared in films, Rhodamine B has a stronger absorption than Oxazine. This leads to an easier detection of some of the features associated with FRET in the samples containing Rhodamine B than in those containing Oxazine. For example, the enhancement of the acceptor PL intensity is only observed in the samples containing nanocrystals mixed with Rhodamine B. ${ }^{1}$ However, Rhodamine B also presents the drawback that it has to be combined with NCs of small enough sizes $(2-2.5 \mathrm{~nm}$ in diameter) in order for the overlap between the NC PL emission and the RhB absorption spectrum to be strong. These

"alphande@tcd.ie; phone + 353-1-608-3281 ; fax +353-1-671-1759 
small NCs have a quantum yield of $15-20 \%$, lower than that reached for bigger NCs. For example, NCs of diameters 4-4.5 $\mathrm{nm}$ have a quantum yield as high as $25-30 \%$ and can be combined with Oxazine to form a FRET pair.

The organization of the paper is as follows. The sample preparations and the experimental setup are first described. For the samples containing CdTe NCs mixed with Rhodamine B, an estimate of the Förster distance is made. Experimental results in support of FRET between CdTe NCs and Rhodamine B or between CdTe NCs and Oxazine are then presented.

\section{METHODOLOGY}

The CdTe nanocrystals are prepared at the University of Hamburg, following a procedure described in ref. 6. They are stabilized by thioglycolic acid and synthesized in aqueous solution by reaction of cadmium perchlorate with $\mathrm{H}_{2} \mathrm{Te}$ gas. Three different solid films are made, composed of either nanocrystals or dyes on their own or nanocrystals mixed with dyes. ${ }^{1}$ In the films containing nanocrystals mixed with Rhodamine B, the ratio between the concentration of acceptors and that of donors, $C_{A} / C_{D}$, is varied between 0.03 and 5 . In those containing nanocrystals mixed with Oxazine, a fixed ratio $C_{A} / C_{D}$ of $\sim 0.3$ is chosen, which corresponds to one of the ratio yielding highly efficient FRET between CdTe NCs and Rhodamine B. ${ }^{1}$ It should also be pointed out that since the donors and the acceptors are contained within the same volume, it is equivalent to speak in terms of the ratio between the concentration of donors and that of acceptors than in terms of the ratio between the amount of acceptors and the amount of donors. As in ref .1, all the measurements described in this paper are performed at room temperature.

\section{EXPERIMENT}

\subsection{FRET between CdTe nanocrystals and Rhodamine B}

A series of experimental results supporting FRET between CdTe nanocrystals and Rhodamine B are presented in ref. 1 . In ref. 1, two families of nanocrystals are studied, those emitting at $525-550 \mathrm{~nm}$ for which the NC PL peak strongly overlaps with the absorption spectrum of Rhodamine B and those emitting at $650 \mathrm{~nm}$ for which there is no overlap

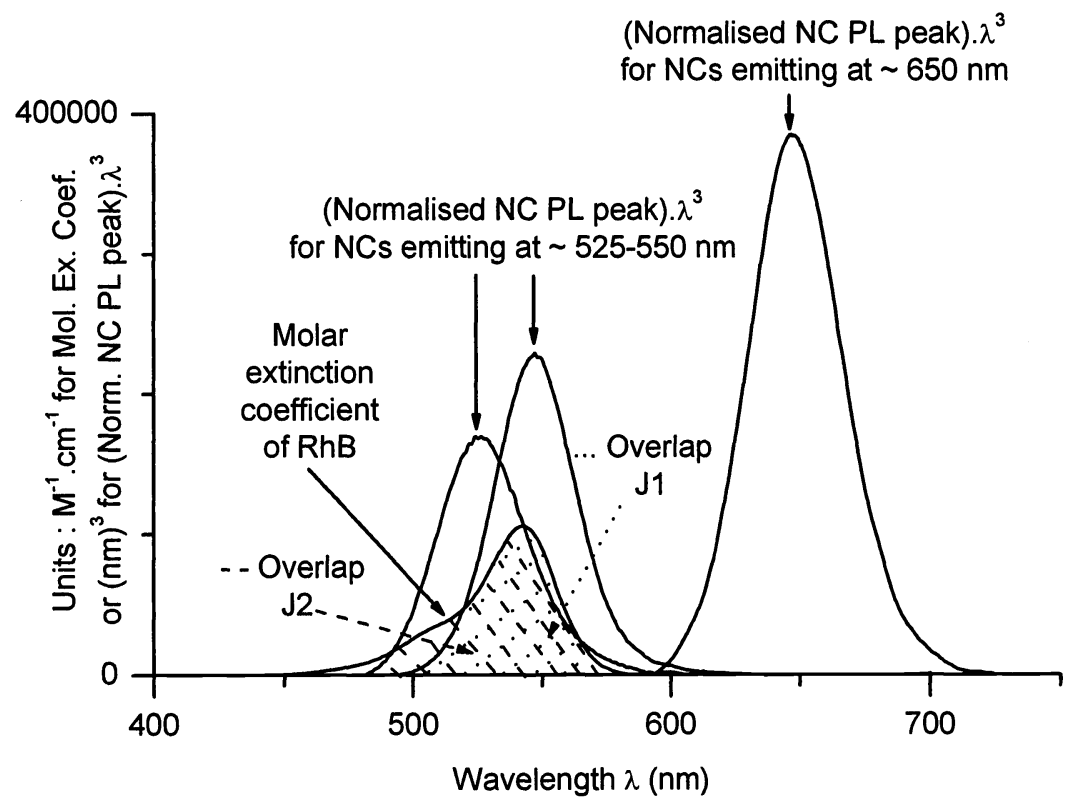

Figure 1: The normalised NC PL peaks multiplied by $\lambda^{3}$ for the NCs emitting at $525-550 \mathrm{~nm}$ or $650 \mathrm{~nm}$ in the units (nm) ${ }^{3}$ and the molar extinction coefficient of Rhodamine B in the units $\mathrm{M}^{-1} . \mathrm{cm}^{-1}$. The overlap integral between the molar extinction coefficient of Rhodamine B and the normalised NC PL peaks multiplied by $\lambda^{3}$ are denoted as J1 and J2 for the nanocrystals emitting at 550 and 525 $\mathrm{nm}$ respectively. 
between the NC PL peak and the absorption spectrum of Rhodamine B (Fig. 1). Since an overlap between the absorption spectrum of the acceptor and the emission spectrum of the donor is one of the conditions required for Förster resonance energy transfer, only the first family of NCs is a good canditate for FRET. In ref. 1, it is reported that FRET is indeed only occurring for the NCs emitting at $525-550 \mathrm{~nm}$. The arguments in support of FRET are based on the increase of the acceptor PL intensity, the decrease of the donor lifetime and the small shift of the NC PLE peak with varying detection wavelength.

In the first experimental section of this paper, the Förster distance is estimated for the NCs emitting at 525-550 nm in order to show that for these NCs, the condition for a highly efficient FRET is fulfilled. The Förster distance is given by the relation ${ }^{1}$

$$
\mathrm{R}_{0}^{6}=8.79 \times 10^{-5}\left\{\mathrm{Q}_{\mathrm{D}} \kappa^{2} \mathrm{n}^{-4} \mathrm{~J}\right\}(\stackrel{0}{\mathrm{~A}})
$$

where $Q_{D}$ is the NC quantum yield, $\kappa^{2}$ is the orientation factor, $n$ is the index of refraction of the surrounding medium and $\mathrm{J}$ is the overlap integral, which is defined as: ${ }^{2}$

$$
J=\int_{0}^{\infty} f_{D}(\lambda) \varepsilon_{A}(\lambda) \lambda^{3} d \lambda
$$

In the above expression, $f_{D}(\lambda)$ is the NC PL peak emission normalised by the area of the NC PL peak and $\varepsilon_{A}(\lambda)$ is the molar extinction coefficient of Rhodamine B. J1 and J2 denote the overlap integrals for the NCs emitting at $550 \mathrm{~nm}$ and $525 \mathrm{~nm}$ respectively. With estimates of $\mathrm{J} 1$ and $\mathrm{J} 2$ of $4.62 .10^{15} \mathrm{M}^{-1} \mathrm{~cm}^{-1}$ and $6.23 .10^{15} \mathrm{M}^{-1} \mathrm{~cm}^{-1}$ respectively, an orientation factor $\kappa^{2}$ assumed to be $2 / 3$, an index of refraction of the surrounding medium assumed to be that of thioglycolic acid (n $=1.505$ ) and a quantum yield of the CdTe nanocrystals assumed to be $2-4 \%$ in films, ${ }^{1}$ a Förster distance of 3-3.5 nm is deduced, which is of the same order of magnitude as that reported for highly efficient FRET between dyes of different species $^{2}$ or NCs of different sizes. ${ }^{5}$

Next, further experimental results in support of FRET between NCs and RhB are presented in Fig. 2. Fig. 2(a) shows the PLE spectrum of the sample containing NCs mixed with RhB, detected at the emission wavelength of the Rhodamine B PL peak emission. ${ }^{1}$ The PLE spectrum shows a peak at $\sim 475 \mathrm{~nm}$, a similar position to that of the absorption peak of the NCs (Fig. 2(b)). The peak at $\sim 475 \mathrm{~nm}$ (Fig. 2(a)) is therefore attributed to NCs.

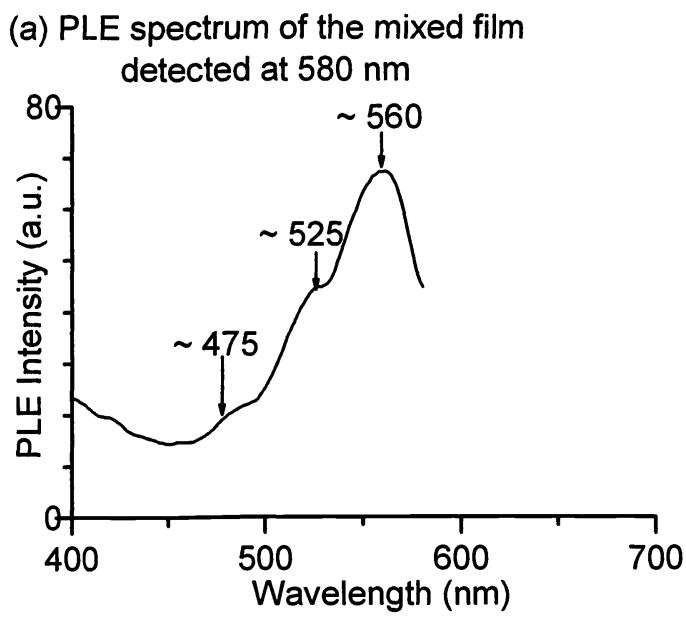

(b) absorption of the mixed film

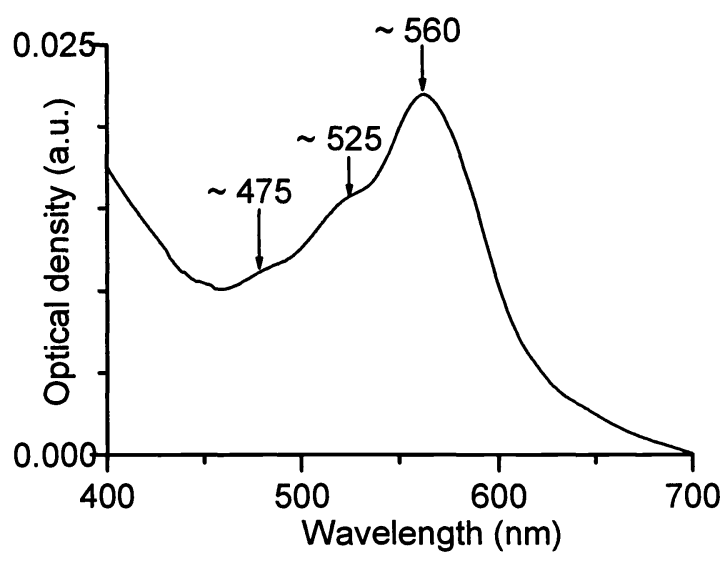

Figure 2: (a) The PLE spectrum of the mixed film detected at the wavelength of the Rhodamine B PL peak emission (580 nm). (b) absorption spectrum of the mixed film. The peak observed at $\sim 475 \mathrm{~nm}$ is attributed to the NCs, while those observed at $\sim 525 \mathrm{~nm}$ and $560 \mathrm{~nm}$ are attributed to Rhodamine B. 
The fact that this peak is observed for a detection wavelength of $580 \mathrm{~nm}$, which is above that of the NC emission $(\sim 525$ $\mathrm{nm}$ ), indicates that it can only arise from the energy transfer between CdTe NCs and Rhodamine B.

More evidence for a FRET process are given by the luminescence decay curves of three different films, containing either NCs on their own or NCs mixed with Rhodamine $B$ in different proportions $\left(C_{A} / C_{D}=1\right.$ or 0.025$)$, which are shown in Fig. 3. For $C_{A} / C_{D}=1$, a faster NC decay curve is observed in the mixed sample compared with that of the sample containing NCs on their own. As already mentioned in ref. 1, this is a signature of FRET. The fact that for $C_{A} / C_{D}=0.025$, the NC decay curve of the mixed film is very similar to that of the sample containing NCs on their own indicates that FRET is only occurring for certain values of the ratio $C_{A} / C_{D}$. It agrees with the results presented in ref. 1, which suggest that for low values of $C_{A} / C_{D}(<0.2)$ or high values of $C_{A} / C_{D}(>5)$, the efficiency of FRET is below 20 \%. More support for the idea that a highly efficient FRET is reached for $0.2<\mathrm{C}_{\mathrm{A}} / \mathrm{C}_{\mathrm{D}}<5$ can be deduced from Fig. 3(b). Fig. 3(b) shows that the strongest ratio between the acceptor and donor PL emission is obtained for $0.2<C_{A} / C_{D}<5$. This suggests that the strongest enhancement of the acceptor PL intensity and therefore the most efficient FRET is also achieved in this region.
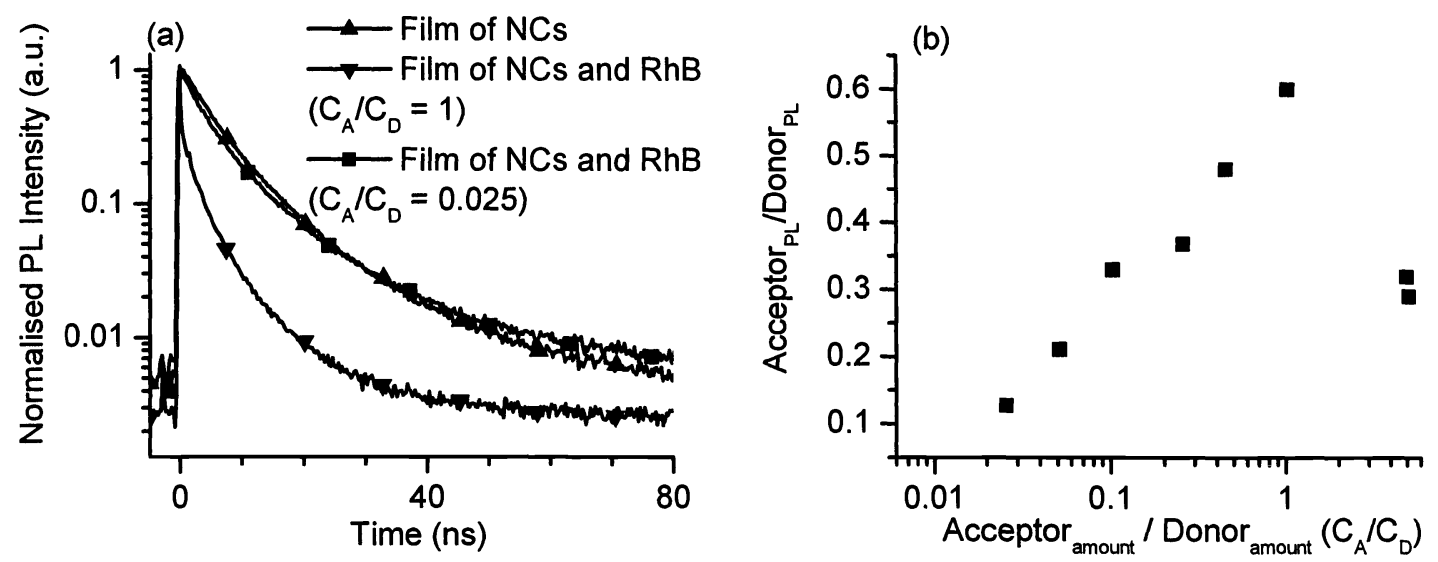

Figure 3: (a) The normalised time dependent PL spectra of the films containing either NCs on their own or NCs mixed with Rhodamine $B$ in different proportions $\left(C_{A} / C_{D}=1\right.$ or 0.025$)$. The decay curved are measured at the wavelength of maximum NC PL intensity. (b) The ratio between the acceptor and donor PL intensity as a function of the ratio between the amount of acceptors and the amount of donors, $\mathrm{C}_{\mathrm{A}} / \mathrm{C}_{\mathrm{D}}$.

\subsection{FRET between CdTe nanocrystals and Oxazine}

In this section, Förster resonance energy transfer between CdTe nanocrystals and Oxazine molecules is studied through lifetime measurements performed on three different types of samples, containing either NCs or Oxazine on their own or NCs mixed with Oxazine. For this study, nanocrystals emitting at $\sim 650 \mathrm{~nm}$ are chosen, in a region where the NC PL peak strongly overlaps with the absorptium spectrum of Oxazine. Figure 4 shows a faster decrease of the NC decay curve in the sample containing NCs mixed with Oxazine than in the sample containing NCs on their own. As for the system of CdTe NCs and Rhodamine B described in section 3.1 and ref. 1, this suggests that FRET between CdTe nanocrystals and Oxazine is indeed occuring.

Comparing the decay curves of the mixed samples in Fig. 3(a) and 4 shows that it is faster in Fig. 4, suggesting that more efficient FRET is reached in the system formed by CdTe NCs and Oxazine than in that formed by CdTe NCs and RhB. Using the same fitting technique as that described in ref. 1, an efficiency of FRET as high as $\sim 60 \%$ is deduced in the system formed by Oxazine and CdTe NCs. 


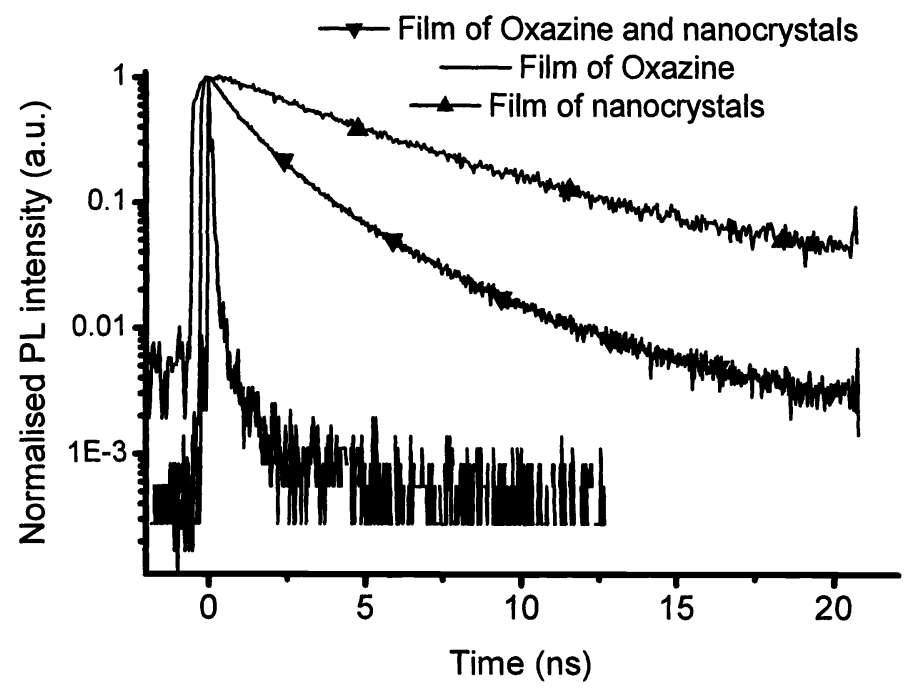

Figure 4: The normalised PL intensities of three different films containing either nanocrystals or Oxazine on their own or nanocrystals mixed with Oxazine.

\section{CONCLUSION}

The main conclusion of this paper is that we have demonstrated highly efficient Förster resonance energy transfer between CdTe nanocrystals and Rhodamine B and between CdTe nanocrystals and Oxazine. One of the limitations of our work to date comes however from the fact that the samples were prepared in mixed solid films. This yields a broad distribution of distances acceptor-donor. Consequently, it is difficult to correlate an estimate of the FRET efficiency with a meaningful distance acceptor-donor. In order to overcome this difficulty, the so-called layer by layer technique will be introduced in the forthcoming experiments. ${ }^{7}$ With this technique, it is possible to control the distance acceptordonor by using one or several layers of polyelectrolyte, which are sandwiched between a layer of dye and a layer of nanocrystals. ${ }^{7}$

\section{REFERENCES}

1. E. Alphandéry, L. M. Walsh, Y. Rakovich, A. L. Bradley, J. F. Donegan, N. Gaponik, Highly efficient Förster resonance energy transfer between CdTe nanocrystals and Rhodamine B in mixed solid films, Chem. Phys. Lett., 388, 100 (2004).

2. B. Van der Meer, G. Coker, S. Y. Simon Chen, Resonance energy transfer: theory and data, VCH Publishers, New-York, 1994.

3. I. L. Medintz, A. R. Clapp, H. Mattoussi, E. R. Goldman, B. Fisher, J. M. Mauro, Self-assembled nanoscale biosensors based on quantum dot FRET donors, Nature materials 2, 630 (2003).

4. S. Wang, N. Mamedova, N. A. Kotov, W. Chen, J. Studer, Antigen/Antibody Immunocomplex from CdTe Nanoparticle Bioconjugates, Nanoletters 2, 817 (2002).

5. C. R. Kagan, C. B. Murray, M. G. Bawendi, Long-range transfer of electronic excitations in close-packed CdSe quantum-dots solids, Phys. Rev. B 54, 8633 (1996).

6. N. Gaponik, D. V. Talapin, A. L. Rogach, K. Hoppe, E. V. Shevchenko, A. Kornowski, A. Eychmüller, H. Weller, Thiol-Capping of CdTe Nanocrystals: An alternative to Organometallic Synthetic Routes, J. Phys. Chem. B 106, 7177 (2002).

7. A. L. Rogach, D. S. Koktysh, M. Harrison, and N. A. Kotov, Layer-by-Layer Assembled Films of HgTe Nanocrystals with Strong Infrared Emission, Chem. Mater. 12, 1526 (2000). 